\title{
Research on EEG-Based Control of Prosthetic Hand
}

\section{Chunsheng $\mathrm{Li}^{1,2}$, a , Haibin Zhao ${ }^{2}$ and Hong Wang ${ }^{2}$}

\author{
${ }^{1}$ School of Electrical Engineering, Shenyang University of Technology, Shenyang 110870, China \\ ${ }^{2}$ School of Mechanical Engineering, Northeastern University, Shenyang 110004, China \\ ali.chunsn@gmail.com
}

\begin{abstract}
Keywords: Electroencephalograph (EEG), wireless control, prosthetic hand, wavelet analysis
\end{abstract}
\begin{abstract}
The wireless control method is implemented between NEU BCI-II brain-computer interface and DR05 humanoid hand in this paper. A user interface with a set of real-time analysis and control methods is developed based on Lab VIEW platform. Wavelet analysis method is embedded in this framework, which extracts the character of alpha rhythm. $86.7 \%$ correct rate is achieved by using this framework to make humanoid hand do six gestures. Electronic disturbance of finger motor is eliminated by adopting wireless method.
\end{abstract}

\section{Introduction}

In recent years, the research of brain-computer interfaces (BCIs), which aims to provide their users communication and control capabilities that do not depend on the brain's normal output channels of peripheral nerves and muscles, arouses more and more interests[1,2]. These signals include visual evoked potential (VEP), slow cortical potentials (SCP) ET al. has been used in BCI systems.

It is well-known that alpha activity in the EEG is dominant in normal individuals during an eyes-closed resting condition, and is suppressed with visual stimulation. This was first reported in the 1930s when several researchers exposed participants to light and noted decreases in occipital alpha activity [3]. Alpha wave's frequency is between 8 and $13 \mathrm{~Hz}$. Kirkup et al. used EEG to control on-off switch by difference of alpha wave between eye opening and eye closing [4]. Wan et al. studied how to use alpha wave to control TV set [5]. This paper studies the power spectrum of alpha wave, and use EEG characters to control prosthetic hand.

As the rapid development of electronic technology and control methods, research on prosthetic hand gets a great improvement [6]. There is millions of amputee in china, and there is about $10 \%$ amputee need prosthetic hand. One key problem is how to control the prosthetic hand. This paper studies how to use EEG alpha wave to control fingers movement of prosthetic hand, and accomplish different gestures. This research will provide a new control method for amputee to help them improve their quality of life.

\section{System Structure}

Wireless prosthetic hand system includes two parts: software and hardware. Software is programmed in LabVIEW, which does data acquisition and analysis. Hardware consists two parts, NEUBCI-II device and DR05 Prosthetic hand. System connection is showed in Fig. 1.

Software. Lab VIEW is a platform and development environment for a visual programming language, which is used widely in control system. The control software can be divided into following blocks: parameter setting, data source management, data acquisition, EEG feature extraction, control mode selection, wireless communication. Based on above implementation, the system can do online data analysis. At the same time, the system can do offline analysis based on data source management block, which make software system very flexible. EEG feature extraction block calls MATLAB Scripts function. Wavelet method is an important tool for analyzing EEG [7, 8, 9]. By MATLAB Scripts, Lab VIEW can connect MATLAB server, and call wavelet function to analyze EEG. Fig. 2 shows the interface. 


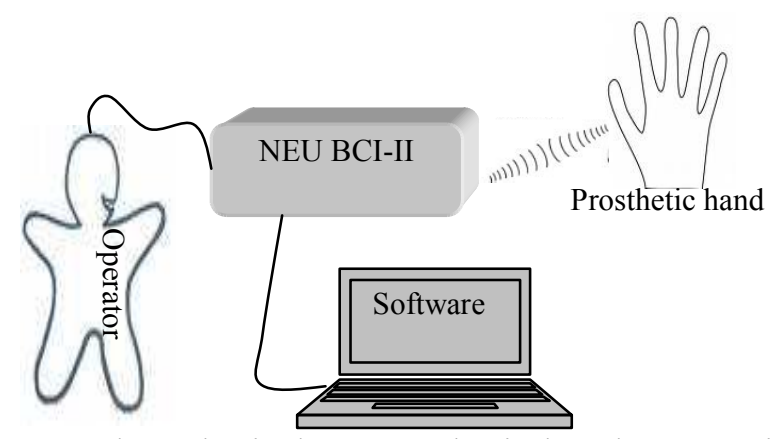

Fig. 1. EEG-based wireless prosthetic hand connections

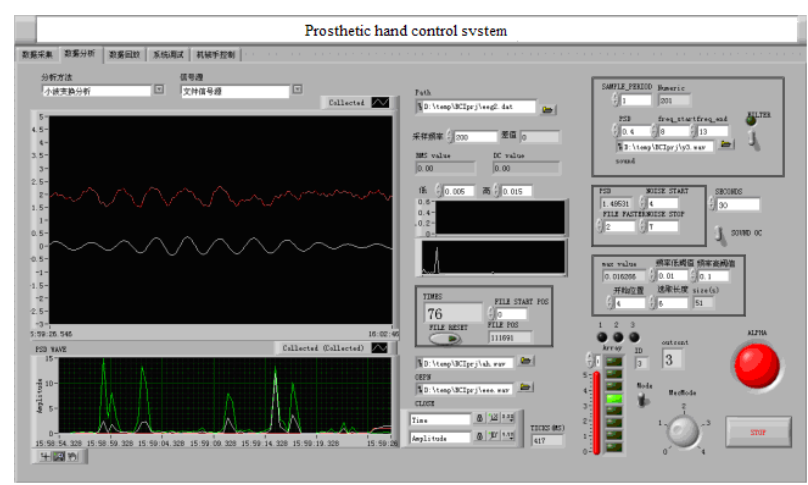

Fig. 2. User interface of the LabVIEW control system

Hardware. NEUBCI-II is a real-time multi-channel EEG device, which provides high gain and CMMR [10]. By adopting optical isolation technology, system noise can be eliminated. The device parameter shows in Table 1.

Table 1 System parameters of NEUBCI-II device

\begin{tabular}{ll}
\hline Parameters & Value \\
\hline gain & $86 \mathrm{~dB}$ \\
band pass & $0.01-30[\mathrm{~Hz}]$ \\
notch & $50[\mathrm{~Hz}]$ \\
channel & 4 \\
type & Differential \\
isolation & Photoelectric \\
sample rate & $100-1000[\mathrm{~Hz}]$ \\
\hline
\end{tabular}

There are 5 fingers in DR05prosthetic hand, and each finger is driven by a separate DC motor. All fingers are coordinated by a microchip, and communicate with NEUBCI-II through wireless module. The prosthetic hand has 5 kinds of working mode, which is described in Table 2 . The user interface can send command to prosthetic hand to switch different mode.

Table 2 Control modes of DR05 prosthetic hand

\begin{tabular}{cl}
\hline Mode & \multicolumn{1}{c}{ Function description } \\
\hline 0 & Self adjustment \\
1 & Absolute mode of finger movement \\
2 & XOR mode of finger movment \\
3 & Single finger mode \\
4 & Parameter setting mode, such as action time, delay et al. \\
\hline
\end{tabular}


The communication between NEUBCI-II and DR05 is based on wireless module. The transfer rate is 2400 bps. Generally, the noise is increased when prosthetic hand motor moves. NEUBCI-II is a high resolution multi-channel device. When prosthetic hand connects it with wires, the noise is increased rapidly. With the wireless connection between NEUBCI-II and prosthetic hand, there is almost no interference in the experiment.

\section{Experiment and Result}

Eight subjects take part in experiment. The $\mathrm{Ag} / \mathrm{AgCl}$ electrode is chosen, and placed on scalp following international 10-20 electrode standard. Occipital (O1) is chosen as signal source and left earlobe (A1) is chosen as reference point. The experiment is accomplished under quiet environment, without special shield. These subjects are asked to keep release as possible, and decrease limb movement. In this experiment, there are 6 indicators, which flash 3 seconds one by one. All subjects should close their eyes when the expected indicator is flashing. Each subject should complete actions 25 times. The control system will detect the variation of alpha wave energy, then it will choose corresponding action according to indicator status. Fig. 3 shows the decomposition of a block EEG data in experiment.

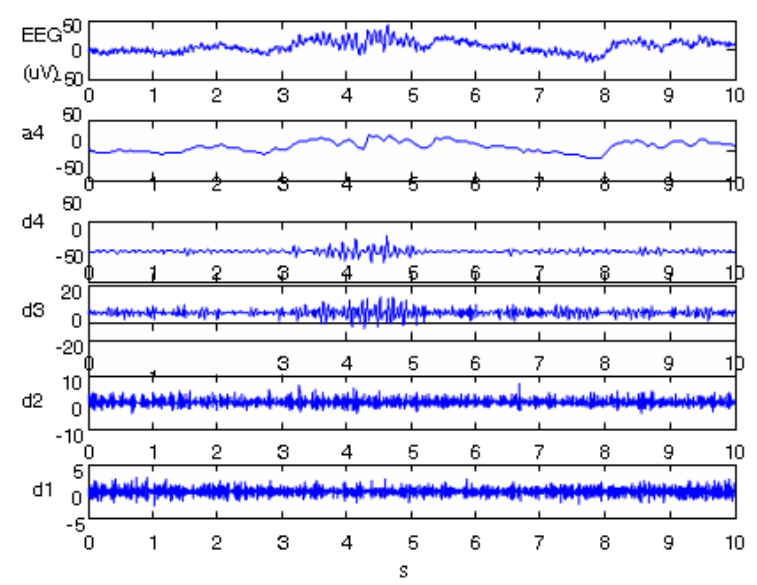

Fig. 3. Wavelet decomposed waveform of EEG

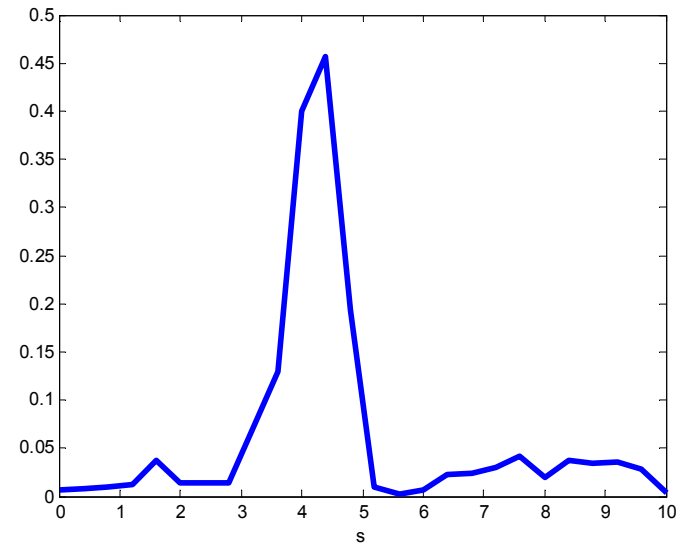

Fig. 4. Variance of EEG energy from 8 to $13 \mathrm{~Hz}$

In Fig. 3, original signal frequency is between 0 and $100 \mathrm{~Hz}$. There are 5 components after decomposition (d1-d4, a4). The frequency of d4is corresponding to $6.25-12.5 \mathrm{~Hz}$, which is increased between 3 and 5 seconds. Fig. 4 is energy variation of d 4 decomposition, which is calculated by wavelet coefficients. It also shows energy increasing obviously between 3 and 5 seconds.

One subject does not accomplish experiment. The research of Craig et al. shows that $10 \%$ normal people's alpha wave can not increase when they close eyes [11, 12]. Hence, this subject is not considered. The experiment result is shown in Table 3.

The correct rate of this EEG-based prosthetic hand experiment is $86.7 \%$, and the average delay is 13.3 seconds. The delay time is composited by three parts: first is the indicator cycle time, second is data acquisition window and analysis time, third is the physiological delay. The indicator cycle time is the main part of delay.

Table 3 Result of prosthetic hand control experiment

\begin{tabular}{ccc}
\hline subjects & movement accuracy & time delay(s) \\
\hline A & $96 \%$ & 13 \\
B & $88 \%$ & 12 \\
C & $72 \%$ & 16 \\
D & $92 \%$ & 13 \\
E & $88 \%$ & 14 \\
F & $84 \%$ & 12 \\
\hline
\end{tabular}




\section{Conclusion}

In EEG-based prosthetic hand experiment, $87.5 \%$ of the operator complete the experiment, and the correct rate is $86.7 \%$. It shows that this research is feasibility and rationality. In the experiment, due to acquisition, physiological reasons, the delay between command and prosthetic hand action is 13.3 seconds. From the view of practice, further improvement needs to be done to reduce the delay time, such as decrease each cycle time, improve control method and so on. In rehabilitation engineering, this study provides a new way to control prosthetic hand for amputees. At the same time, this research will also improve their quality of life. In addition, wireless communication is introduced into this system, and electronic disturbance of finger motor is eliminated.

\section{References}

[1] J.R. Wolpaw, Brain-computer interface research comes of age: traditional assumptions meet emerging realities, J Mot Behav. 42(2010) 351-353.

[2] D.J. McFarland, W.A. Sarnacki, J.R. Wolpaw, Should the parameters of a BCI translation algorithm be continually adapted? J Neurosci Methods, 199(2011) 103-107.

[3] J.R. Wolpaw, N. Birbaumer, W.J. Heetderks, D.J. McFarland, P.H. Peckham, Brain-computer interface technology: a review of the first international meeting, IEEE T. Neur. Sys. Reh. 8 (2000) 164-173.

[4] L. Kirkup, A. Searle, A. Craig, P. McIsaac, G. Larsen, Three methods compared for detecting the onset of alpha wave synchronization following eye closure, Physiol. Meas. 19 (1998) 213-224.

[5] B.K. Wan, H.Z. Qi, L. Zhao, B.J. Chen, B. Dhakal, Q. Chen, Remote control experiments of brain-computer interface based on alpha waves in electroencephalography, Journal of Tianjin University, 39 (2006) 978-984.

[6] L. Jiang, H. Liu, Real time force optimization algorithm of multi-fingered grasp, Chinese Journal of Mechanical Engineering, 43 (2007) 144-149.

[7] Z.Y. Wang, H. Wang, Y.N. Li, X. Wang, Wavelet analysis of evoked electroencephalogram (EEG) in brain-computer interface, Journal of Northeastern University(Natural Science), 26 (2005) 546-549.

[8] B.H. Yang, G.Z. Yan, R.G. Yan, H. Sun, Feature extraction for EEG-based brain-computer interfaces by wavelet packet best basis decomposition, J. Neural Eng. 3 (2006) 251-256.

[9] F.S. Yang, Engineering Analysis and Application of Wavelet Transformation, Science Press, Beijing, 1999.

[10] C.S. Li, H. Wang, Wavelet transform for on-off switching BCI device, IFMBE Proceedings of 7th Asian-Pacific Conference on Medical and Biological Engineering, Beijing, 2008, 363-365.

[11] W. Hardle, T. Gasser, P. Bacher, EEG-responsiveness to eye opening and closing in mildly retarded children compared to a control group, Biol. Psychol.18 (1984) 185-199.

[12] A. Craig, P. McIsaac, Y. Tran, L. Kirkup, A. Searle. Alpha wave reactivity following eye closure: a potential method of remote hands free control for the disabled, Technology and Disability, 10 (1999) 187-194. 